\title{
Synthesis of antimicrobial silver nanoparticles through a photomediated reaction in an aqueous environment
}

This article was published in the following Dove Press journal:

International Journal of Nanomedicine

19 January 2016

Number of times this article has been viewed

\author{
Rafał Banasiuk ${ }^{1, *}$ \\ Joanna E Frackowiak ${ }^{2, *}$ \\ Marta Krychowiak' \\ Marta Matuszewska' \\ Anna Kawiak' \\ Magdalena Ziabka ${ }^{3}$ \\ Zofia Lendzion-Bielun ${ }^{4}$ \\ Magdalena Narajczyk ${ }^{5}$ \\ Aleksandra Krolicka' \\ 'Department of Biotechnology, \\ Intercollegiate Faculty of \\ Biotechnology, University of Gdańsk \\ and Medical University of Gdańsk, \\ ${ }^{2}$ Department of Pathophysiology, \\ Medical University of Gdańsk, \\ Gdańsk, ${ }^{3}$ Faculty of Materials Science \\ and Ceramics, Department of \\ Ceramics and Refractories, AGH- \\ University of Science and Technology, \\ Kraków, ${ }^{4}$ Institute of Chemical and \\ Environment Engineering, West \\ Pomeranian University of Technology, \\ Szczecin, ${ }^{5}$ Faculty of Biology, \\ Laboratory of Electron Microscopy, \\ University of Gdańsk, Gdańsk, Poland \\ *These authors contributed equally \\ to this work
}

Correspondence: Aleksandra Krolicka Intercollegiate Faculty of Biotechnology, University of Gdańsk and Medical University of Gdańsk, Laboratory of Biologically Active Compounds, 24 Kladki, 80-822 Gdańsk, Poland

Tel +48585236305

Fax +48585236426

Email aleksandra.krolicka@biotech. ug.edu.pl

\begin{abstract}
A fast, economical, and reproducible method for nanoparticle synthesis has been developed in our laboratory. The reaction is performed in an aqueous environment and utilizes light emitted by commercially available $1 \mathrm{~W}$ light-emitting diodes $(\lambda=420 \mathrm{~nm})$ as the catalyst. This method does not require nanoparticle seeds or toxic chemicals. The irradiation process is carried out for a period of up to 10 minutes, significantly reducing the time required for synthesis as well as environmental impact. By modulating various reaction parameters silver nanoparticles were obtained, which were predominantly either spherical or cubic. The produced nanoparticles demonstrated strong antimicrobial activity toward the examined bacterial strains. Additionally, testing the effect of silver nanoparticles on the human keratinocyte cell line and human peripheral blood mononuclear cells revealed that their cytotoxicity may be limited by modulating the employed concentrations of nanoparticles.
\end{abstract}

Keywords: antimicrobial activity, green synthesis, nanocubes, nanospheres

\section{Introduction}

Silver nanoparticles (AgNPs) are known for their broad antimicrobial activity toward multiple species of microbes including multidrug-resistant bacteria and fungi. ${ }^{1,2}$ This makes them an invaluable alternative for traditional antibiotic treatment. Recent reports suggest that silver and gold nanoparticles can form spontaneously in the natural environment from dissolved organic matter. ${ }^{3}$ This fact provides valuable insight into the nature of the reaction, which is successfully employed in "green" synthesis of nanoparticles described in many publications. Several other reports suggest the importance of the chloride ion ${ }^{4}$ for the reaction to take place. While polyol synthesis of $\mathrm{AgNPs}^{4-6}$ provides reproducible results, it is necessary to develop methods which not only are faster and environmentally friendly, but also possible to upscale, allowing to meet the growing demand for this industry. The possibility of incorporating nanoparticles into fabrics, polymers, paints, electronics, diagnostic methods, etc requires specific parameters of the nanoparticles such as size, shape, dispersion, and solubility in various solvents. Nanoparticle synthesis may be performed using various methods including polysaccharide reduction, irradiation (microwave, ${ }^{7}$ ultraviolet, ${ }^{8}$ laser, ${ }^{9}$ gamma radiation, ${ }^{10}$ artificial sunlight ${ }^{11}$ ), the Tollens method, and biological synthesis (using extracts from plants, bacteria, fungi, as well as peptides and live organisms). ${ }^{12}$ These approaches allow to obtain the desired product, however, they may considerably benefit from several alterations. In spite of an enormous amount of research related to this topic, new methods as well as further modifications of existing protocols continue to be developed. Several issues need to be addressed today including time, energy, as well as the cost and quality of reagents 
required for the process. While in bench-scale production these factors are minor, their relevance is indisputable when considering industrial-scale production. The paper describes a simple, scalable, one-pot synthesis of nanoparticles, which is performed in an aqueous environment and utilizes commercially available $1 \mathrm{~W}$ light-emitting diodes (LEDs) $(\lambda=420 \mathrm{~nm})$ as the catalyst. The method does not require the use of nanoparticle seeds or toxic chemicals. The process is rapid and ecologically friendly as the irradiation process is completed within 10 minutes. By employing specific reaction parameters, we predominantly obtained spherical or cubic nanoparticles which demonstrated antimicrobial activity. Considering the fact that the synthesized AgNPs may ultimately be intended for medical applications such as wound healing, we tested the obtained AgNPs regarding their effect on human keratinocytes to ensure safety of their use. We also considered the possibility of AgNPs penetrating the skin and entering the bloodstream as demonstrated by many drugs. Therefore, the effect of AgNPs on human blood cells was examined using peripheral blood mononuclear cells (PBMCs) obtained from healthy volunteers.

\section{Materials and methods}

\section{LED light source characteristics}

Irradiation was performed using a lamp designed and constructed in our laboratory. The lamp exhibited the following characteristics: number of LEDs per radiator plate: 5; LED power (per LED): $1 \mathrm{~W}$; radiator plate dimensions: $70 \times 70 \mathrm{~mm}$; diagonal between LEDs placed on opposite edges of the plate: $50 \mathrm{~mm}$; voltage (per LED): $3.2 \mathrm{~V}$; current (per LED): $350 \mathrm{~mA}$; luminosity (per LED): 20-25 lm; LED viewing angle: $140^{\circ}$; wavelength $\left(\lambda_{\max }\right)$ : various between 395 and $620 \mathrm{~nm}$; height between light source and reaction vessel: $60 \mathrm{~mm}$. Only one type of LED was used in a given experiment.

\section{Synthesis of silver nanoparticles}

All experiments were performed in $250 \mathrm{~mL}$ borosilicate bottles. The final volume of the reaction mixture was $200 \mathrm{~mL}$. Compounds being the source of chloride ions were added prior to the 10 -minute irradiation period. Irradiation was carried out with no other light source present. The use of defined concentrations of chemicals and compounds enabled the formation of specific types of nanoparticles (Table 1).

\section{Sample concentration}

Prior to testing the samples for in vitro activity and characterizing the scanning electron microscopy (SEM)/transmission electron microscopy (TEM) parameters, the nanoparticles were filtered through a $0.2 \mu \mathrm{m}$ mixed cellulose-ester syringe filter and concentrated by centrifugation (15,000 rcf, 30 minutes). The supernatant was discarded and the sediment was suspended in $2 \mathrm{~mL}$ of deionized water.

\section{Characterization of nanoparticles by TEM}

Samples were placed on grids (Sigma-Aldrich, St Louis, MO, USA) coated with a $2 \%$ collodion solution (SigmaAldrich). Samples absorbed to the surface were examined using a Philips CM100 electron microscope at $80 \mathrm{kV}$ (FEI Company, Eindhoven, the Netherlands).

\section{Characterization of nanoparticles by SEM}

The morphology of AgNPs was investigated by SEM (Nova Nano SEM 200; FEI Company). Imaging was carried out in low vacuum in the secondary electron mode using the HELIX detector. Tests were performed using accelerating voltage of 5-10 keV and 3-4 spots, depending on the measurement capability selected for individual samples.

\section{Determination of synthesis efficacy}

The efficacy of synthesis was established by measuring the concentration of residual silver ions present after the reaction was completed and this value was compared with the state before synthesis. The obtained product was centrifuged at 15,000 rcf for 30 minutes. The supernatant was tested for silver content and then discarded. Silver content was determined by inductively coupled plasma-atomic emission spectroscopy. The study was performed using the Perkin Elmer Optima 5300 DV emission spectrometer. A calibration curve was prepared using the standard $1,000 \mu \mathrm{g} / \mathrm{mL}$ silver

Table I Concentrations of particular compounds used for synthesis of AgNPs (per $100 \mathrm{~mL}$ )

\begin{tabular}{lllll}
\hline Glycine AgNPs & \multicolumn{3}{c}{ PVP AgNPs } & SDS AgNPs \\
\hline $\mathrm{AgNO}_{3}$ & $\mathrm{I} \mathrm{mM}$ & $\mathrm{AgNO}$ & $4 \mathrm{mM}$ & $\mathrm{AgNO}_{3}$ \\
$\mathrm{PVP}$ & $150 \mathrm{mg}$ & $\mathrm{PVP}$ & $200 \mathrm{mg}$ & SDS \\
Glycine & $14 \mathrm{mM}$ & $5.25 \% \mathrm{NaClO}$ & $0.5 \mathrm{~mL}$ & $5.25 \% \mathrm{NaClO}$ \\
I M Tris- $\mathrm{HCl}(\mathrm{pH} \mathrm{8.5)}$ & $20 \mu \mathrm{L}$ & & & \\
\hline
\end{tabular}

Abbreviations: AgNPs, silver nanoparticles; PVP, polyvinylpyrrolidone; SDS, sodium dodecyl sulfate. 
dissolved in nitric acid. Analysis was carried out using the emission wavelength of silver at $328.068 \mathrm{~nm}$.

\section{Study of antimicrobial and antifungal susceptibility}

Antimicrobial and antifungal activity of AgNPs was determined using the Broth Microdilutions Method according to guidelines provided by the Clinical and Laboratory Standards Institute ${ }^{13}$ regarding the Staphylococcus aureus strain ATCC 13420, the Pseudomonas aeruginosa strain PAK originally isolated by D Bradley (Memorial University of Newfoundland, St John's, Canada), the Escherichia coli clinical isolate 950 (Provincial Hospital of Gdańsk, Poland), and the Candida albicans clinical isolate 17150/2010 (Provincial Hospital of Gdańsk). Initial inoculum was obtained by densitometry (DensiMeter II; EMO, Brno, Czech Republic) from dilutions of overnight cultures $\left(37^{\circ} \mathrm{C}\right)$ in the brain-heart infusion medium to a final bacterial concentration of $5 \times 10^{5}$ colony-forming units (cfu/mL). Minimal inhibitory concentration (MIC) values were defined as the lowest concentration of AgNPs, at which no visible growth of the pathogen was observed after a 24 -hour incubation period at $37^{\circ} \mathrm{C}$.

\section{The effect of nanoparticles on bacteria established by TEM}

An overnight culture of $P$. aeruginosa (strain PAK) grown on the brain-heart infusion broth was diluted to $5 \mathrm{McF}$ arland $\left(1.5 \times 10^{9} \mathrm{cfu} / \mathrm{mL}\right)$ with the use of a densitometer (DensiMeter II; EMO); $4.0 \mu \mathrm{L}$ of nanoparticles were added (equivalent to $\mathrm{MIC}=2$ ) to $3 \mathrm{~mL}$ of the bacterial suspension and the following time points were examined: the initial time of nanoparticle addition to the bacteria as well as after 3, 6, 9, and 12 hours of incubation with AgNPs at $37^{\circ} \mathrm{C}$. Following the incubation period, bacteria were centrifuged, washed twice with phosphate-buffered saline, fixed with $2.5 \%$ glutaraldehyde (Polysciences), and then with $1 \%$ osmium tetroxide (Polysciences, Warrington, PA, USA). Following ethanol dehydration, bacteria were embedded in Epon 812 resin (Sigma-Aldrich). ${ }^{14}$ Ultrathin sections $(55 \mathrm{~nm})$ were cut on the ultramicrotome Leica UC7, while lead citrate and uranyl acetate were added as contrasting agents. TEM studies were performed using the Philips CM100 microscope.

\section{Cytotoxicity of AgNPs studied on the human keratinocyte cell line model}

The cytotoxicity assay was performed on the immortalized human keratinocyte cell line HaCaT (CLS order no 300493) using the method described previously. ${ }^{15}$ The experiment tested the following concentrations of AgNPs: 0.1, 0.2, 0.3, 0.5 , and $1.0 \mu \mathrm{L} / \mathrm{mL}$ of medium (which correspond to 0.2 , $0.4,0.6,1.0$, and $2.0 \mu \mathrm{g}$ nanoparticles $/ \mathrm{mL}$ ).

\section{Testing for cytotoxicity of AgNPs on human PBMCs}

The study included four healthy volunteers (mean age = $27.5 \pm 1.5$ years), two women, and two men. The participants were informed about the purpose of the study and provided written consent. The study was approved by the Bioethical Committee for Scientific Research at the Medical University of Gdańsk, Poland. Twenty $\mathrm{mL}$ of venous blood from each individual was drawn into sterile tubes containing the anticoagulant agent ethylenediaminetetraacetic acid. Blood was diluted with Dulbecco's Phosphate-Buffered Saline, $\mathrm{Ca}^{2+} / \mathrm{Mg}^{2+}$ free (Gibco, Paisley, UK) and PBMCs were isolated by centrifugation through a Histopaque-1077 gradient. The buffy coat consisting of PBMCs was collected and washed three times with Dulbecco's Phosphate-Buffered Saline. The isolated PBMCs were suspended in Roswell Park Memorial Institute-1640 medium supplemented with $10 \mathrm{U} / \mathrm{mL}$ penicillin, $1 \mu \mathrm{g} / \mathrm{mL}$ streptomycin, $10 \%$ fetal calf serum, and $2 \mathrm{mM}$ L-glutamine. The reagents mentioned were purchased from Sigma-Aldrich, unless mentioned otherwise. The PBMC suspension was placed on a 24-well cell culture polystyrene flat bottom plate (Corning Incorporated, Corning, NY, USA) at a concentration of 2 million cells $/ 2 \mathrm{~mL}$ of medium in each well. The following volumes of AgNPs were added to cells suspended in $2 \mathrm{~mL}$ of medium: $0.02,0.1,0.2,0.5,1,2$, and $4 \mu \mathrm{L}$ (which correspond to $0.04,0.2,0.4,1,2,4$, and $8 \mu \mathrm{g}$ ). Cells were incubated at $37^{\circ} \mathrm{C}, 5 \% \mathrm{CO}_{2}$ for 3 hours, after which their viability was assessed using the phycoerythrin Annexin V Apoptosis Detection Kit I (BD-Biosciences, San Diego, CA, USA) according to the manufacturer's instructions.

\section{Flow cytometry data analysis}

Cytometric analysis was performed using the FACScan flow cytometer (Becton Dickinson, Franklin Lakes, NJ, USA). Lymphocytes and monocytes which constitute the PBMC population were gated according to their forward scatter and side scatter characteristics, which were determined by their size and granularity, respectively. Analysis of fluorescence emitted by 7-aminoactinomycin D (7-AAD) and by the fluorochrome phycoerythrin conjugated with Annexin V indicated cell death within the gated populations. Annexin V is a calcium-dependent protein characterized by high affinity for the phospholipid phosphatidylserine normally present on the inner surface of the membrane. The staining procedure 
allows for the identification of early apoptotic cells due to the fact that membranes of such cells lose their asymmetry, leading to the translocation of phosphatidylserine to the outer surface of the membrane where it is bound by Annexin V. The viability probe 7-AAD is capable of entering dead cells through their damaged membranes and staining DNA, whereas viable cells with intact membranes are not permeable to this compound. Staining performed using both Annexin V and 7-AAD allows to distinguish between dead cells being a result of late apoptosis or necrosis from those undergoing early apoptosis. Cells positive for Annexin V alone are defined as early apoptotic with their membrane still intact, while Annexin V- and 7-AAD-positive cells are described as dead resulting from a necrotic pathway or undergoing late apoptosis. The obtained data were analyzed using the Cyflogic software, version 1.2.1 (Perttu Terho and CyFlow Ltd.).

\section{Statistical analysis}

Values were expressed as means \pm standard deviation of at least three independent experiments. Differences between control and one-treated samples were analyzed by one-way analysis of variance with Tukey's post hoc tests. $P \leq 0.05$ was considered as statistically significant in each experiment.

\section{Results}

\section{Optimization of the protocol for AgNP synthesis}

Laboratory synthesis of AgNPs was performed under conditions resembling those which occur in the natural environment. We selected the lysogeny broth (LB) bacterial medium as a model of organic matter naturally present in the environment. We chose this particular material on the basis of our observations which indicated its capability of AgNP production when spiked with silver to a final concentration of $1 \mathrm{mM} \mathrm{AgNO}$ and exposed for a defined time to artificial or natural light at room temperature. We determined whether the process requires the whole light spectrum or rather a specific band. As our main objectives included an economic approach as well as the reproducibility of the results regardless of the site performing the procedure, we chose the commercially available LEDs. We tested various wavelengths emitted by $1 \mathrm{~W}$ LEDs, ranging from 395 to $620 \mathrm{~nm}$. The studied mixture consisted of $10 \mathrm{~mL} \mathrm{LB}$ and $300 \mathrm{mg}$ polyvinylpyrrolidone (PVP) (molecular weight: $40,000 \mathrm{M}$ ) dissolved in $200 \mathrm{~mL}$ deionized water spiked with $\mathrm{AgNO}_{3}$ to a final concentration of $1 \mathrm{mM}$ (Table 1). The duration of the reaction was assessed experimentally based on the change in color (colorless or slightly yellow to $\mathrm{red} /$ dark red) and inductively coupled plasma-atomic emission spectroscopy analysis. We tested various irradiation periods which ranged from 1 to 30 minutes. Best results were obtained when the sample was irradiated using $420 \mathrm{~nm}$ light. Any other wavelength failed to promote synthesis of nanoparticles leaving the reaction mixture a white-to-cream colored colloid due to the formation of insoluble silver salts. The reaction reached the highest efficiency of $96.3 \%$ 10 minutes after its initiation, which was determined by testing for the presence of residual silver. These results were subsequently validated by TEM. No change in the morphology of nanoparticles was observed during the course of the reaction. The concentration of nanoparticles was the single affected parameter. Subsequent experiments were focused on the simplification of the reaction mixture. By using the LB medium composed of defined constituents instead of a plant extract of unknown composition, we were able to study individual compounds required for the reaction. The reaction was performed in $250 \mathrm{~mL}$ borosilicate glass bottles containing $200 \mathrm{~mL}$ of $1 \mathrm{mM} \mathrm{AgNO}_{3}$ and $300 \mathrm{mg}$ of PVP unless stated otherwise. Although subjected to irradiation, the reaction mixture alone failed to produce nanoparticles. The complete LB medium as well as its peptone fraction, when used at the final concentration present in the LB medium, both yielded similar results. However, when used separately, the individual amino acid components of the LB medium did not lead to nanoparticle formation. We discovered that the addition of chloride ions was required for the initiation of the reaction. Further studies revealed that glycine, alanine, and leucine present within the peptone fraction were key amino acids leading to the formation of AgNPs. Glycine was tested in subsequent experiments as its addition produced the highest yield of nanoparticles. The source of the chloride ion seemed to be irrelevant for the initiation of the reaction as our research involving hydrochloric acid, sodium chloride $(\mathrm{NaCl})$, potassium chloride, and Tris- $\mathrm{HCl}(\mathrm{pH} 8.5)$ yielded similar results. In order to determine whether other types of chlorine compounds may provide the chloride ion, we tested sodium hypochlorite $(\mathrm{NaClO})$, sodium chlorate $\left(\mathrm{NaClO}_{3}\right)$, sodium perchlorate $\left(\mathrm{NaClO}_{4}\right)$, and trichloroacetic acid $\left(\mathrm{C}_{2} \mathrm{HCl}_{3} \mathrm{O}_{2}\right)$. The addition of $\mathrm{NaClO}$ changed the solution from colorless to violet as a result of irradiation. This was due to the dissolution of the $\mathrm{ClO}^{-}$ion, which we confirmed by obtaining similar results using $\mathrm{NaCl}$ with hydrogen peroxide. Next, we verified whether replacing the capping agent with another may influence the properties presented by the AgNPs, allowing them to acquire superior antimicrobial activity. 


\section{TEM and SEM characterization of nanoparticles}

TEM images (Figure 1) revealed that nanoparticles synthesized using glycine were spherical, whereas those using $\mathrm{NaClO}$ were cubical. SEM (Figure 1) showed that the size of nanoparticles synthesized in the presence of glycine and with the use of PVP as a capping agent was highly irregular in size compared with those synthesized with the use of $\mathrm{NaClO}$ and that they demonstrated a tendency of clump formation. We performed experiments which assessed the impact of compound selection on the size and shape of the nanoparticles. We found that PVP concentrations higher than $200 \mathrm{mg} / 100 \mathrm{~mL}$ caused the aggregation of nanoparticles, while concentrations higher still (400 mg/100 mL) resulted in the loss of their shape, which
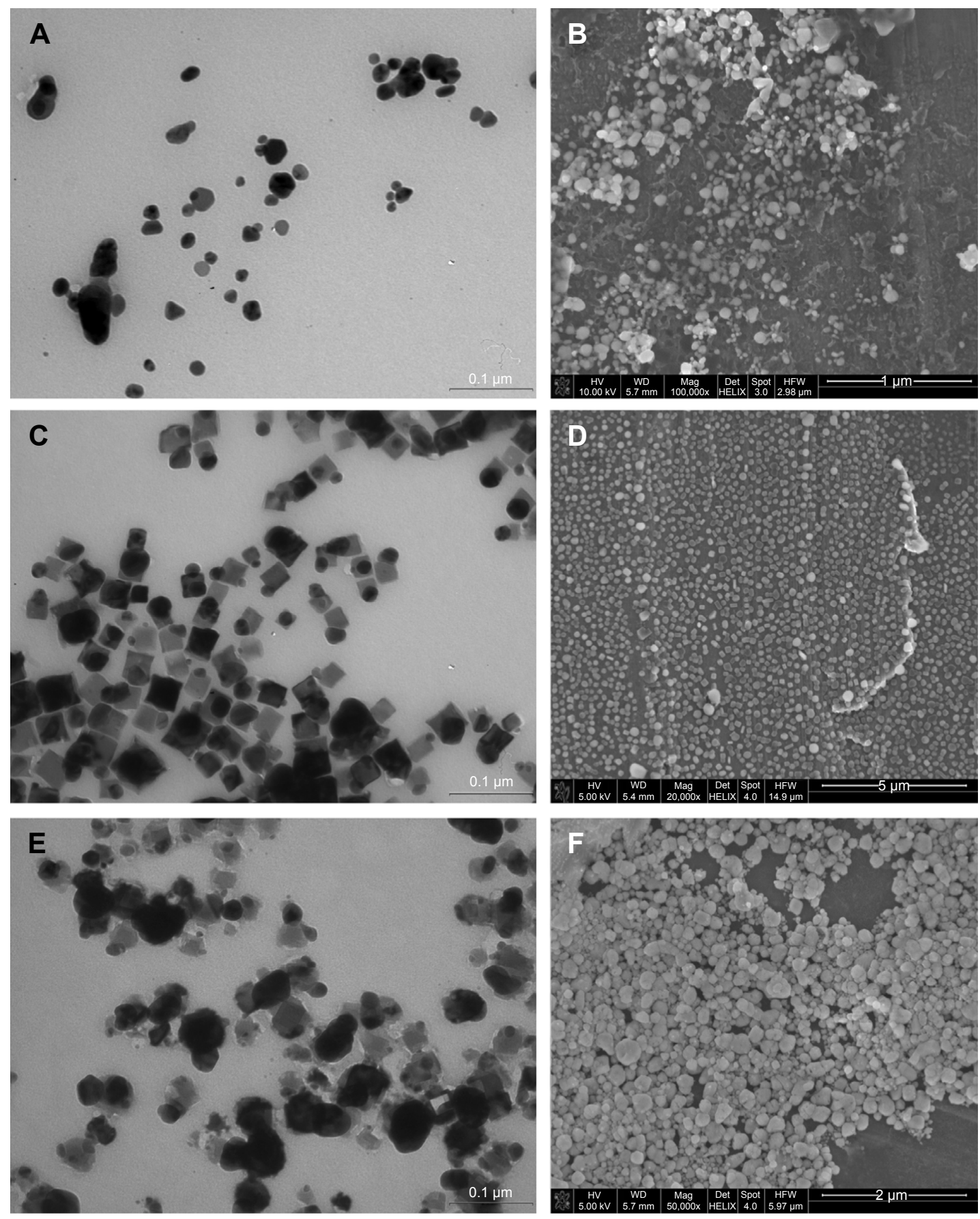

Figure I TEM and SEM images of silver nanoparticles synthesized using (A) TEM, (B) SEM glycine, (C) TEM, (D) SEM PVP, (E) TEM, (F) SEM SDS. Abbreviations: PVP, polyvinylpyrrolidone; SEM, scanning electron microscopy; SDS, sodium dodecyl sulfate; TEM, transmission electron microscopy. 

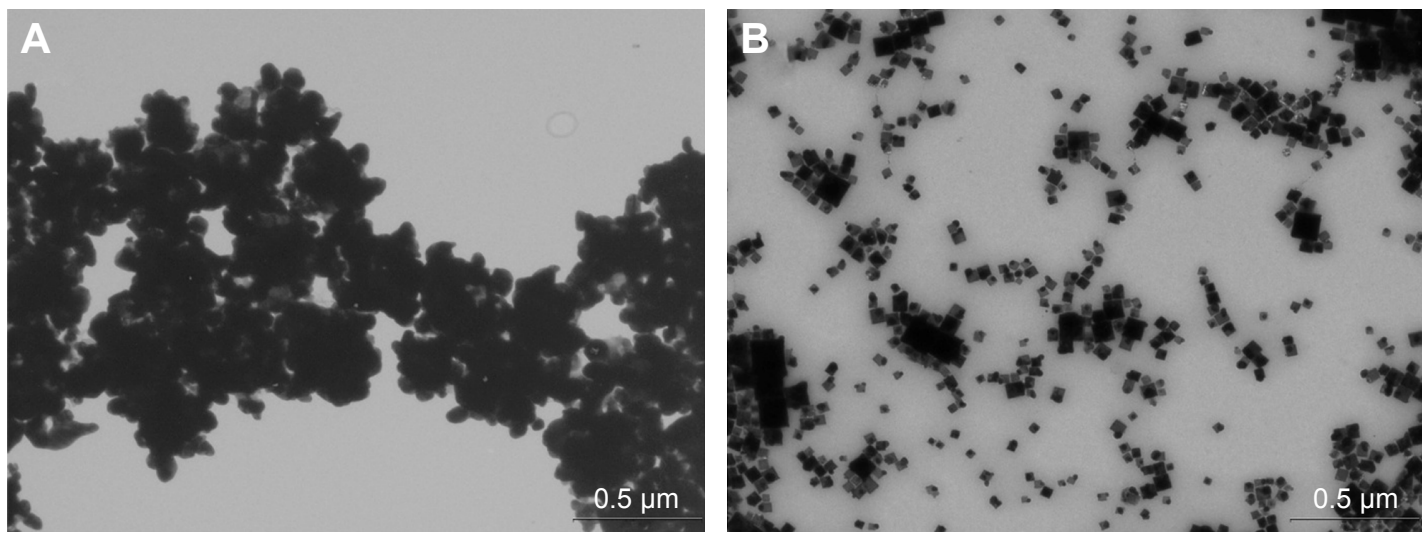

Figure 2 TEM images of silver nanoparticles synthesized using I mM AgNO, 1 mL 5\% NaClO per $100 \mathrm{~mL}$ and (A) $400 \mathrm{mg}$ PVP per $100 \mathrm{~mL}$, (B) $200 \mathrm{mg}$ PVP per $100 \mathrm{~mL}$; irradiation time 10 minutes, light wavelength $420 \mathrm{~nm}$.

Abbreviations: PVP, polyvinylpyrrolidone; TEM, transmission electron microscopy.

was most apparent regarding square-shaped nanoparticles (Figure 2). PVP levels between 100 and $200 \mathrm{mg} / 100 \mathrm{~mL}$ were optimal for obtaining nanoparticles below $50 \mathrm{~nm}$ in diameter. Size variation of sodium dodecyl sulfate (SDS)-capped nanoparticles was far greater than that of PVP-capped nanoparticles and it ranged between 0.6 and $100 \mathrm{~nm}$. SDS concentration above $2.5 \mathrm{mM}$ prompted the selective production of small nanoparticles as we observed a positive correlation between the amount of SDS added and the count of particles which were $0.6 \mathrm{~nm}$ in diameter (Figure 3). In certain conditions (Table 1), the structures show relatively high uniformity in size and can be further fractionated using centrifugation.

\section{Testing antimicrobial and antifungal susceptibility}

On the basis of TEM and SEM analysis, the PVP-capped AgNPs were selected for further studies of their possible

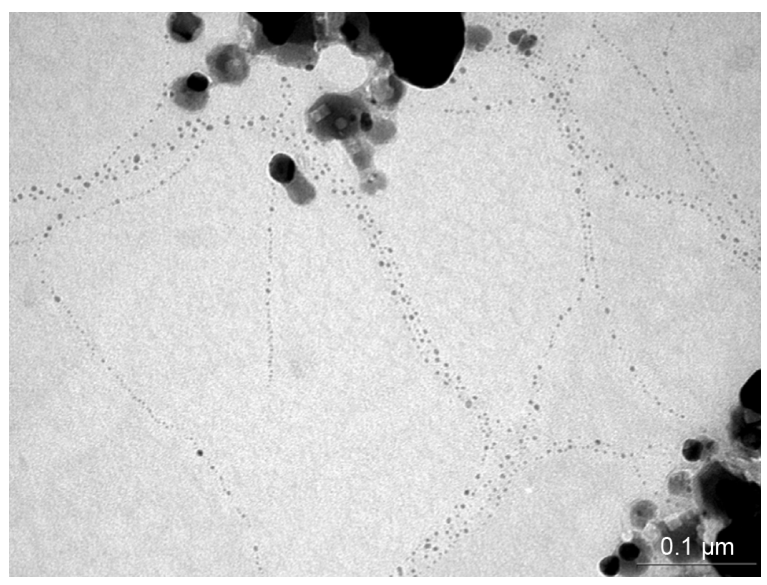

Figure 3 TEM images of silver nanoparticles synthesized using $2 \mathrm{mM} \mathrm{AgNO}_{3}, 5 \mathrm{mM}$ SDS, $0.5 \mathrm{~mL} 5 \% \mathrm{NaClO}$ per $100 \mathrm{~mL}$; irradiation time 10 minutes, light wavelength $420 \mathrm{~nm}$.

Abbreviations: SDS, sodium dodecyl sulfate;TEM, transmission electron microscopy. antimicrobial and antifungal activity (Figure $1 \mathrm{C}$ and D). The obtained nanoparticles exhibited strong antimicrobial properties against the tested Gram-positive and Gram-negative bacterial strains. MIC values for nanoparticles were as follows: $1.0 \mu \mathrm{g}$ PVP-capped AgNPs/mL for P. aeruginosa, E. coli, and C. albicans and $2.0 \mu \mathrm{g}$ PVP-capped AgNPs $/ \mathrm{mL}$ for $S$. aureus. Each compound used for synthesis was individually tested for its antimicrobial and antifungal activity; none however exhibited any activity within the concentration range used.

\section{Testing for interactions between bacteria and nanoparticles using TEM}

Subsequent studies regarding the mode of action demonstrated by the obtained nanoparticles were performed on the $P$. aeruginosa strain PAK selected as the model organism. The bacterial suspension was treated with square-shaped nanoparticles at a concentration corresponding to the double minimal inhibitory concentration. After incubation, the sample was fixed and examined by TEM (Figure 4). Surprisingly, the images showed no disruption in the outer membrane and instead revealed condensation of bacterial DNA.

\section{Cytotoxicity of AgNPs toward the human keratinocyte cell line}

As PVP-capped AgNPs exhibited strong antimicrobial activity, making them potentially applicable in the treatment of burn wounds, it was necessary to test whether they could be considered safe for human cells. Thus, the human keratinocyte cell line HaCaT was treated with PVP-capped AgNPs and analyzed using the tetrazolium salt thiazolyl blue (MTT) assay in order to assess viability. The assay revealed that the highest concentrations ( 2 and $4 \mu \mathrm{g} / \mathrm{mL}$ ) 

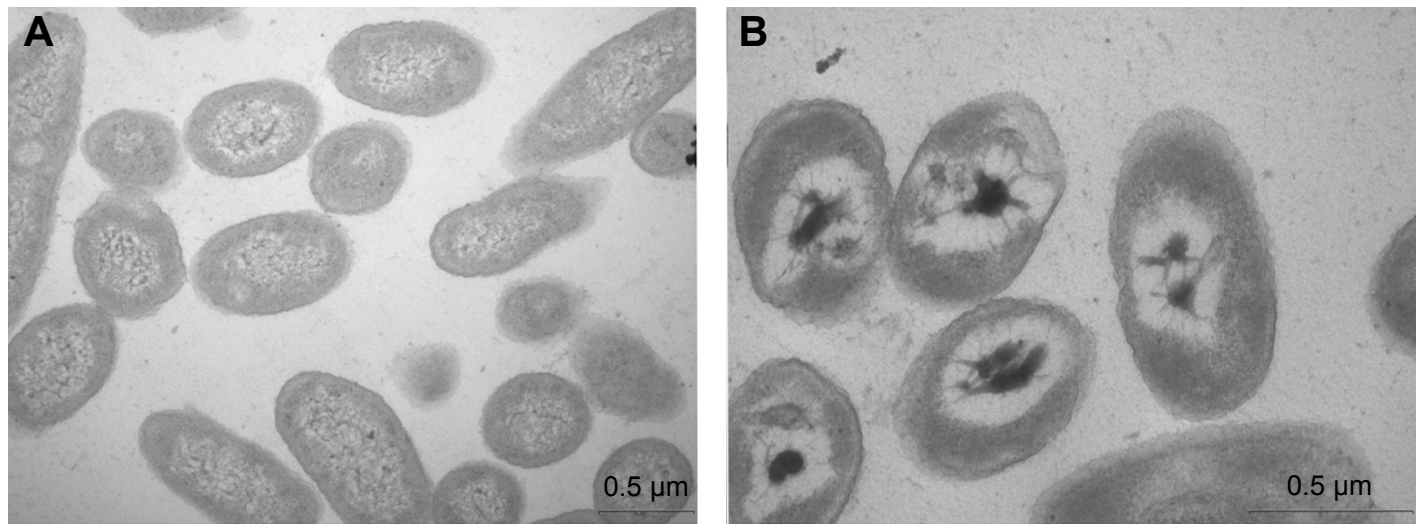

Figure 4 TEM images of Pseudomonas aeruginosa PAK: (A) control, (B) after 9 hours incubation with 2 MIC PVP-capped nanoparticles. Abbreviations: 2 MIC, double minimal inhibitory concentration; PVP, polyvinylpyrrolidone; TEM, transmission electron microscopy.

of AgNPs used in this study exhibited cytotoxic effects on human keratinocytes resulting in $63 \%$ and $88 \%$ decrease in their viability (Figure 5).

\section{Testing for cytotoxicity of AgNPs toward human PBMCs}

Among the studied concentrations, $0.02,0.1$, and $0.2 \mu \mathrm{g}$ of $\mathrm{AgNPs} / \mathrm{mL}$ had no effect on the viability of PBMCs. The mean percentage of Annexin V-negative and 7-AAD-negative cells in the control was determined as $96.4 \%$ for lymphocytes and $94.8 \%$ for monocytes. These values were similar for samples treated with concentrations up to $0.2 \mu \mathrm{g}$ of $\mathrm{AgNPs} / \mathrm{mL}$, where $95.4 \%$ of lymphocytes and $94.5 \%$ of monocytes were viable. Figure $6 \mathrm{~A}$ and $\mathrm{B}$ shows representative dot plots of the control as well as cells treated with $0.2 \mu \mathrm{g}$ of $\mathrm{AgNPs} / \mathrm{mL}$ obtained from selected experiments. These cells demonstrated normal morphology as indicated by their forward scatter and side scatter characteristics and two distinct lymphocyte and monocyte populations were clearly visible. However, increasing the

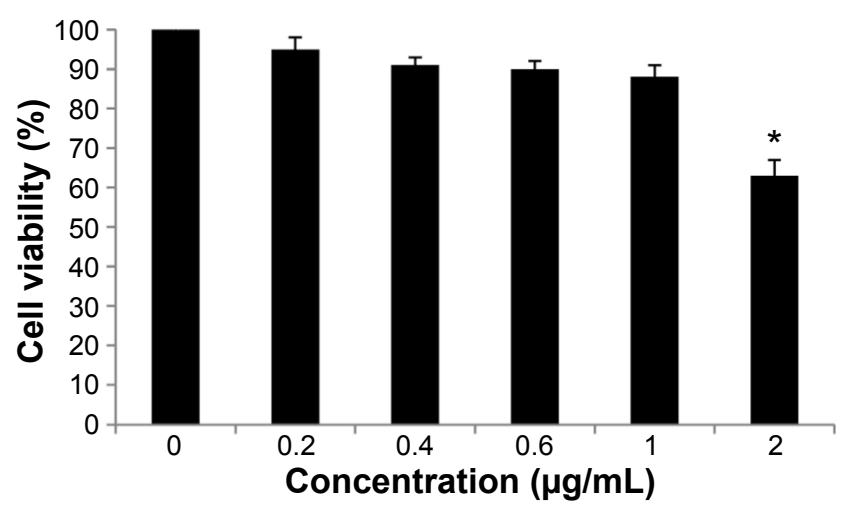

Figure 5 Cytotoxic effect of various concentrations of AgNPs on human keratinocytes $(n=3)$.

Notes: Data were analyzed by one-way ANOVA with Tukey's post hoc tests; $P \leq 0.05$ ( $\left.^{*}\right)$ indicates differences between the control and one-treated cells. Toxicity was assessed after 24 hours of incubation at $37^{\circ} \mathrm{C}$ in the dark.

Abbreviations: AgNPs, silver nanoparticles; ANOVA, analysis of variance. concentration to $0.5 \mu \mathrm{g}$ AgNPs/mL (Figure 6C) resulted in the dramatic change in their morphology, cells were decidedly smaller, and more granular, which was expressed in their forward scatter and side scatter characteristics. Further changes involved their membrane, resulting in an Annexin V-positive staining for $12.75 \%$ or both Annexin V- and 7-AAD-positive staining for $84.4 \%$ of cells. This demonstrated a dramatic decline in cell viability, as the majority was found to be either early apoptotic or necrotic/late apoptotic. Raising the concentration of AgNPs to $2 \mu \mathrm{g}$ AgNPs/mL increased the cytotoxic effect further (Figure 6D).

\section{Discussion}

Our method shows a new approach for the synthesis of AgNPs, significantly reducing time (10 minutes per batch) and energy consumption (by using LEDs). As opposed to other methods, which use light source in order to influence the shape of nanoparticles, our method uses light as a main catalyst in the reaction. ${ }^{16,17}$ With the continuous expansion of the AgNP market, this new method of synthesis may reduce the environmental impact concerning waste and chemical usage. While most "green" synthesis methods based on plant extracts, ${ }^{18}$ bacterial, ${ }^{19}$ and fungal ${ }^{20}$ supernatants are indisputably environmentally friendly, their reproducibility is strongly dependent on the conditions required for the cultivation of these organisms. Due to many factors involved, including biological variation, the results may differ between individual reactions. This fact renders most "green" synthesis routes unsuitable for commercial use. The possibility of using cheap, readily available reagents instead of biological matrices allows for easy replication of the process on a larger scale. Moreover, the procedure can be further automatized to finally require no human interaction other than maintenance of the production line. While it is possible to use various solvents for the production of nanoparticles, water alone enables 

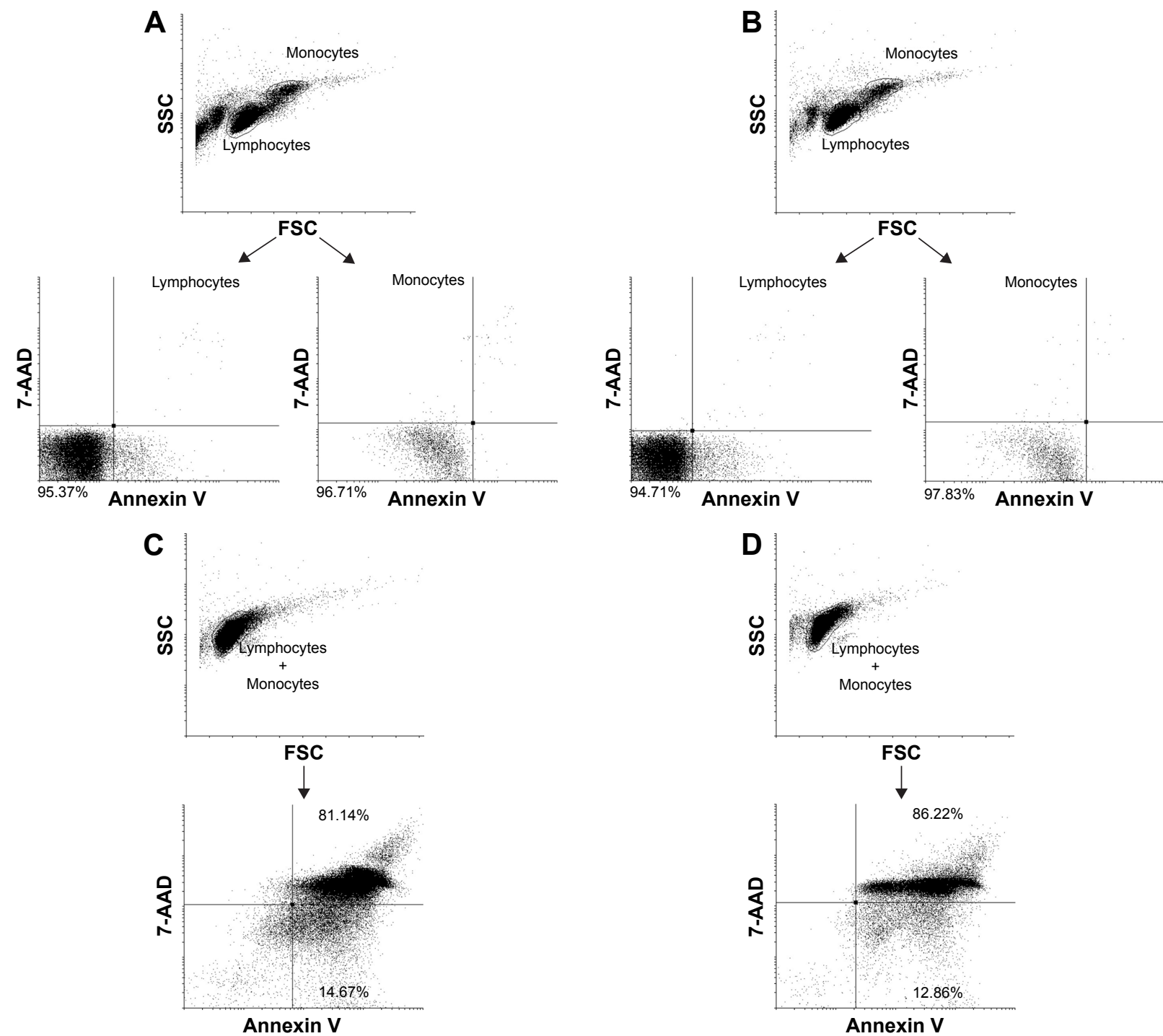

Figure 6 Cytotoxic effects of AgNPs on human PBMCs.

Notes: Untreated control (A) and PBMCs treated with $0.2 \mu \mathrm{g} / \mathrm{mL}$ of AgNPs (B) consist of two distinct populations (lymphocytes and monocytes), which predominantly are Annexin $\mathrm{V}$ and 7-AAD negative, indicating their viability. PBMCs treated with AgNPs at a concentration of $0.5 \mu \mathrm{g} / \mathrm{mL}(\mathbf{C})$ and higher $(2 \mu \mathrm{g} / \mathrm{mL}[\mathbf{D}])$ demonstrate a change in their morphology as smaller and more granular cells are observed, expressed in FSC and SSC. Changes also include their membrane, resulting in either an Annexin V-positive or both Annexin $V$ and 7-AAD-positive staining for the majority of cells, demonstrating a high death percentage. The cytometric analysis shows two representative experiments.

Abbreviations: 7-AAD, 7-aminoactinomycin D; AgNPs, silver nanoparticles; FSC, forward scatter; PBMCs, peripheral blood mononuclear cells; SSC, side scatter.

their direct use in applications such as hydrogel wound dressings or latex paints, allowing our method to be used in various fields. Additionally, we observed that our PVP-capped nanoparticles may easily be dispersed in methanol or acetic acid, which is desired in applications requiring a nonaqueous environment such as sanitary silicones. A further advantage of our method is the fact that the equipment needed for the reaction can be constructed from commercially available elements with minimal effort. This is essential should our AgNPs be applied in bandages used for treating burn wound infections. We demonstrated that AgNPs in concentrations up to 0.2 and $2 \mu \mathrm{g} / \mathrm{mL}$ are not cytotoxic toward human PBMCs and keratinocytes, respectively. Burn wounds are one of the most common, highly debilitating injuries leading to the occurrence of an immunologically weakened region deprived of humoral and cellular response. Consequently, this area is particularly susceptible to infection. If left untreated, this condition is potentially fatal as it may ultimately weaken the entire body. Despite progress in the treatment of burn wounds, including the immediate removal of burned tissue, skin grafting or the introduction of new antibiotic treatment, microbiological infections are the leading cause of death among burn patients. ${ }^{21}$ Burn wound infections as well as various other types of infections are commonly treated by 
commercial drugs. Unfortunately, major disadvantages of this approach include severe side effects and the development of drug-resistant bacteria. The introduction of antibiotics in medicine has nearly entirely eradicated multiple species of pathogenic microorganisms. However, the ubiquitous use of these drugs resulted in the emergence of multiple drugresistant bacterial species. $^{22}$

The intracellular action of AgNPs is based on their interaction with particular compounds, which contain either a phosphorous or sulfur group, that is, mainly nucleic acids and proteins. ${ }^{23}$ The presence of AgNPs in the cytoplasm triggers the bacterial response to toxic substances such as silver ions, leading to DNA condensation. ${ }^{24,25}$ Through binding to thiol groups, AgNPs also affect proteins, causing the disruption of their structure and leading to their inactivation. This affects multiple types of proteins that are essential for the proper function of bacteria, leading to the deactivation of the ribosome complex, deactivation of the active transport protein, inhibition of enzymes involved in the tricarboxylic acid cycle, and ultimately resulting in their death. ${ }^{2}$ Due to its antiseptic properties, silver has been used for centuries in the treatment of various infections. It has been demonstrated that AgNPs efficiently eliminate the Gram-positive bacteria $S$. aureus as well as its multiple drug-resistant species. ${ }^{26}$ Moreover, AgNPs promote burn wound healing and consequently improve the skin's appearance by preventing scar formation. Although the cost of AgNPs as well as the conditions they may cause when inappropriately used (such as argyria) are an evident disadvantage, their beneficial properties are unquestionable. Therefore, establishing innovative and cost-effective means of incorporating AgNPs into novel therapies shows great promise. It ought to be stressed that nanocrystalline silver dressings for the treatment of burn wounds, such as Acticoat (Westaim Biomedical Inc., Fort Saskatchewan, Alberta, Canada) are already commercially available. These materials exhibit superior bactericidal activity compared with silver nitrate and prevent the development of nosocomial infections in burn wounds. Research using pigs established that nanocrystalline silver dressings accelerate wound healing and reduce inflammation. It has been demonstrated that nanocrystalline silver dressings release silver ions $\left(\mathrm{Ag}^{+}\right)$and atoms of silver (" $\mathrm{Ag}^{0}$ clusters") from their surface, which determines their antibacterial activity. ${ }^{27} \mathrm{We}$ are currently establishing the formulation of a hydrogel wound dressing which best accommodates our nanoparticles.

\section{Conclusion}

Here, we described a simple, one-pot method for the synthesis of nanoparticles in an aqueous environment. The reaction uses water as the solvent and does not require toxic additives such as sodium borohydride. As a result, the synthesized nanoparticles may be used without prior purification in applications such as wound dressings, disinfectants, and water-based paints. Furthermore, our studies showed that the MIC values of our AgNPs were not cytotoxic toward human keratinocytes and PBMCs. Our research points to the basic factors to be considered for further development of "green" nanoparticle chemistry.

\section{Acknowledgments}

This work was partially realized using the resources of the Cellular Function and Pathology Imaging Network of the Medical University and the University of Gdańsk.

Publication costs were covered by the EU FP7 project MOBI4Health, GA.316094. The funders had no role in study design, data collection and analysis, decision to publish, or preparation of the manuscript.

\section{Disclosure}

The authors report no conflicts of interest in this work.

\section{References}

1. Kim J, Kuk E, Yu K, et al. Antimicrobial effects of silver nanoparticles. Nanomedicine. 2007;3:95-101.

2. Rai M, Deshmukh S, Ingle A, Gade A. A silver nanoparticles: the powerful nanoweapon against multidrug-resistant bacteria. $J$ Appl Microbiol. 2012;112:841-852.

3. Yin Y, Liu J, Jiang G. Sunlight-induced reduction of ionic Ag and Au to metallic nanoparticles by dissolved organic matter. ACS Nano. 2012;6: 7910-7919.

4. Wiley B, Herricks T, Sun Y, Xia Y. Polyol synthesis of silver nanoparticles: use of chloride and oxygen to promote the formation of single-crystal, truncated cubes and tetrahedrons. Nano Lett. 2004;4: 1733-1739.

5. Byeon J, Kim Y. A novel polyol method to synthesize colloidal silver nanoparticles by ultrasonic irradiation. Ultrasonics Sonochem. 2012; 19:209-215.

6. Siekkinen AR, McLellan JM, Chen J, Xia Y. Rapid synthesis of small silver nanocubes by mediating polyol reduction with a trace amount of sodium sulfide or sodium hydrosulfide. Chem Physics Lett. 2006;432: 491-496.

7. Hu B, Wang S, Wang K, Zhang M, Yu S. Microwave-assisted rapid facile "green" synthesis of uniform silver nanoparticles: self-assembly into multilayered films and their optical properties. J Phys Chem C. 2008;112:11169-11174.

8. Darroudi M, Ahmad M, Zak A, Zamiri R, Hakimi M. Fabrication and characterization of gelatin stabilized silver nanoparticles under UV-light. Int J Mol Sci. 2011;12:6346-6356.

9. Matos RAD, Cordeiro TDS, Samad RE, et al. Synthesis of silver nanoparticles using agar-agar water solution and femtosecond pulse laser irradiation. Colloids Surf A Physicochem Eng Aspects. 2013;423:58-62.

10. Long D, Wu G, Chen S. Preparation of oligochitosan stabilized silver nanoparticles by gamma irradiation. Radiation Phys Chem. 2007;76: 1126-1131.

11. Pietrobon B, Kitaev V. Photochemical synthesis of monodisperse sizecontrolled silver decahedral nanoparticles and their remarkable optical properties. Chem Mater. 2008;20:5186-5190. 
12. Sharma VK, Yngard RA, Lin Y. Silver nanoparticles: green synthesis and their antimicrobial activities. Adv Colloid Interface Sci. 2009;145: 83-96.

13. Wayne PA. Methods for dilution antimicrobial susceptibility tests for bacteria that grow aerobically; approved standard, Ninth Edition. CLSI document M07-A9. Clinical and Laboratory Standards Institute; 2012.

14. Le AT, Le TT, Nguyen VQ, et al. Powerful colloidal silver nanoparticles for the prevention of gastrointestinal bacterial infections. Adv Nat Sci: Nanosci Nanotechnol. 2012;3:045007.

15. Krychowiak M, Grinholc M, Banasiuk R, et al. Combination of silver nanoparticles and Drosera binata extract as a possible alternative for antibiotic treatment of burn wound infections caused by resistant Staphylococcus aureus. PLoS One. 2014;9(12):e115727.

16. Callegari A, Tonti D, Chergui M. Photochemically grown silver nanoparticles with wavelength-controlled size and shape. Nano Lett. 2003; 3:1565-1568

17. Tang B, Xu S, Hou X, et al. Shape evolution of silver nanoplates through heating and photoinduction. ACS Appl Mater Interfaces. 2013;5: 646-653.

18. Ghaffari-Moghaddam M,Hadi-Dabanlou R, Khajeh M, Rakhshanipour M, Shameli K. Green synthesis of silver nanoparticles using plant extracts. Korean J Chem Eng. 2014;31:548-557.

19. Shahverdi A, Minaeian S, Shahverdi H, Jamalifar H, Nohi A. Rapid synthesis of silver nanoparticles using culture supernatants of Enterobacteria: a novel biological approach. Process Biochem. 2007;42: 919-923.
20. Karthik L, Kumar G, Kirthi A, Rahuman A, Rao K. Streptomyces sp LK3 mediated synthesis of silver nanoparticles and its biomedical application. Bioprocess Biosyst Eng. 2014;37:261-267.

21. Murray CK. Infections in burns. J Trauma. 2007;62:S73.

22. Tsymbal A, Pechenizkiy M, Puuronen S, Shifrin M, Alexandrova I. Knowledge discovery in microbiology data: analysis of antibiotic resistance in nosocomial infections. In: International Workshop on Current Aspects of Knowledge Management in Medicine, KMM05, in conjunction with the 3rd International Conference on Professional Knowledge Management: Experiences and Visions, Kaiserslautern, Germany, Lecture Notes in Computer Science, Springer; 2005.

23. Lara HH, Garza-Treviño EN, Ixtepan-Turrent L, Singh DK. Silver nanoparticles are broad-spectrum bactericidal and virucidal compounds. J Nanobiotechnol. 2011;9:30.

24. Li WR, Xie XB, Shi QS, et al. Antibacterial effect of silver nanoparticles on Staphylococcus aureus. Biometals. 2011;24:135-141.

25. Morones JR, Elechiguerra JL, Camacho A, et al. The bactericidal effect of silver nanoparticles. Nanotechnology. 2005;16:2346-2353.

26. Panacek A, Kvitek L, Prucek R, et al. Silver colloid nanoparticles: synthesis, characterization, and their antibacterial activity. J Phys Chem B. 2006;110:16248-16253.

27. Dunn K, Edwards-Jones V. The role of Acticoat ${ }^{\mathrm{TM}}$ with nanocrystalline silver in the management of burns. Burns. 2004;30(Suppl I):S1-S9.
International Journal of Nanomedicine

\section{Publish your work in this journal}

The International Journal of Nanomedicine is an international, peerreviewed journal focusing on the application of nanotechnology in diagnostics, therapeutics, and drug delivery systems throughout the biomedical field. This journal is indexed on PubMed Central, MedLine, CAS, SciSearch $®$, Current Contents ${ } /$ Clinical Medicine,

\section{Dovepress}

Journal Citation Reports/Science Edition, EMBase, Scopus and the Elsevier Bibliographic databases. The manuscript management system is completely online and includes a very quick and fair peer-review system, which is all easy to use. Visit http://www.dovepress.com/ testimonials.php to read real quotes from published authors. 